\title{
Prevalencia de sífilis materna y evaluación de una prueba diagnóstica rápida en Cochabamba, Bolivia
}

\author{
Nayrah Villazón-Vargas ${ }^{1}$, Carlos J Conde-G lez ${ }^{1}$, \\ Luis Juárez-Figueroa ${ }^{1}$, Felipe U ribe-Salas ${ }^{1,2}$. \\ Evaluation of a rapid diagnostic test \\ to assess the prevalence of maternal \\ syphilis in Bolivia
}

Background: Syphilis is still a public health problem in Latin America and the Caribbean. Therefore maternal screening is of great importance for public health. Aim: To estimate the prevalence of maternal syphilis and to evaluate a rapid diagnostic test for the disease among Bolivian women. Material and methods: A total of 489 women delivering in a Public Hospital in Cochabamba, Bolivia, answered a questionnaire about socioeconomic and reproductive characteristics and access to health care services. Syphilis was diagnosed by using RPR and FTA-ABS tests. The rapid test Determine syphilis TP was evaluated taking as reference FTA-ABS test. Results: Twenty two women (4.5\%) were positive both for RPR and FTA-ABS tests and fifty $(10.2 \%)$ were positive for the rapid diagnostic test and FTA-ABS. Compared with FTA-ABS, the sensitivity and specificity of the rapid test were $98 \%$ and $99.8 \%$, respectively. Only $15 \%$ of women that had at least one antenatal care visit had a syphilis screening test and $40 \%$ of the women in whom the test was performed, did not receive its results. Increasing age, low socioeconomic level and a history of a sexually transmitted disease were independently associated with syphilis infection. Conclusions: The magnitude of syphilis infection among the studied women was still high in Cochabamba. The rapid test showed excellent overall performance for maternal syphilis screening. Few women of all attended in the hospital had a screening syphilis test and half of those screened were not aware of the results. Maternal syphilis affected older women of low socioeconomic level and with a history of a sexually transmitted disease (Rev Méd Chile 2009; 137: 515-21).

(Key words: Health services; Public health; Syphilis serodiagnosis)

Recibido el 16 de septiembre, 2008. Aceptado el 7 de enero, 2009.

Trabajo financiado parcialmente por el Instituto Nacional de Salud Pública de México. Las pruebas Determine TP fueron donadas por Abbott Laboratories.

${ }^{1}$ Instituto Nacional de Salud Pública, Centro de Investigaciones sobre Enfermedades Infecciosas, Cuernavaca, Morelos, México. ${ }^{2}$ El Colegio de la Frontera Norte, Región Noreste, Piedras Negras, Coahuila, México.

Correspondencia a: Dr. Felipe Uribe-Salas. El Colegio de la Frontera Norte. Calles Progreso y Hacienda № 503, Col. Burócratas, Piedras Negras, Coahuila, México. Código Postal 26020.

Teléfono: 52 (878) 782 5020. E mail: fjusalas@gmail.com

ARtíCULO DE 
$\mathrm{A}_{\mathrm{w}}^{\mathrm{p}}$ pesar de que la sífilis materna aún representa un problema de Salud Pública en América Latina y el Caribe, poca atención se le ha proporcionado en comparación con la dedicada a la prevención y control de la infección materna por el virus de inmunodeficiencia humana (VIH) ${ }^{1}$. La importancia del estudio de la sífilis materna estriba en que dicha infección tiene consecuencias patológicas para el producto de la concepción durante el embarazo y después del nacimiento ${ }^{2,3}$.

Sobre la magnitud de sífilis materna en América Latina y el Caribe tenemos que información suministrada a la Organización Panamericana de la Salud por los programas nacionales mostró en el año 2002 que la prevalencia global en la región fue de 3,1\% con variaciones entre 1\% en Perú y 6,2\% en Paraguay ${ }^{4}$. Estudios realizados en clínicas de atención de parto en diferentes países de América Latina y el Caribe mostraron prevalencias discrepantes que variaron entre los casos extremos de $0,38 \%$ en México y $11 \%$ en Haitíi 5 . En este contexto cabe resaltar que en Bolivia se ha reportado una prevalencia global de sífilis materna de 4,3\%13 pero existe el antecedente en el Hospital Germán Urquidi (HGU) de Cochabamba, de una prevalencia de $10,9 \% 13$, estando esta última cifra entre las más altas reportadas en la región.

Se ha propuesto que los siguientes factores de riesgo han estado asociados a la infección por sífilis materna ${ }^{14}$ : indicadores de nivel socioeconómico bajo como ingreso y escolaridad y características de comportamiento individual como consumo de drogas ilegales, consumo de tabaco y alcohol, así como la presencia de múltiples parejas sexuales. No obstante, los mayores riesgos de sífilis congénita son un limitado número de consultas prenatales y una baja frecuencia en realización de pruebas diagnósticas de sífilis durante dichas consultas. Así, el funcionamiento de los servicios de salud juega un papel importante en el diagnóstico oportuno de sífilis materna y por lo tanto de sífilis congénita ${ }^{15}$ por lo cual dicho funcionamiento debe ser evaluado como un factor de riesgo más.

Durante los últimos 5 años ha habido un auge en el uso de pruebas rápidas para el diagnóstico de sífilis en países en desarrollo ${ }^{16}$. La razón de ello estriba en que la realización de dichas pruebas no requiere de equipo de laboratorio ni entrenamiento sofisticado en comparación de las pruebas como VDRL o RPR. La prueba Determine syphilis TP
(Determine) tiene un principio inmunocromatográfico que se presenta en una tirilla reactiva que detecta anticuerpos contra antígenos de $\mathrm{T}$ pallidum. Al agregar la muestra sanguínea problema, ésta fluye a través de la almohadilla de la tira hasta que se mezcla con un conjugado coloide con selenio y antígenos de $\mathrm{T}$ pallidum inmovilizados teniendo un resultado en aproximadamente quince minutos ${ }^{17}$. Un estudio realizado para evaluar el comportamiento de seis diferentes pruebas rápidas mostró que Determine tuvo un excelente desempeño con valores altos de sensibilidad $(97,2 \%)$ y de especificidad $(94,1 \%)^{18}$. En otro estudio en que se utilizó Determine se obtuvo una sensibilidad alta que varió entre $96,8 \%$ y $100 \%$ al ser aplicada en tres grupos de población con prevalencias de sífilis entre 1,3\% y 38,7\% en México, mientras que la especificidad varió entre $95,3 \%$ y $100 \% 19$, lo cual muestra una alta reproducibilidad de la prueba en diferentes poblaciones y una utilidad potencial para realizar pruebas de tamizaje.

En noviembre de 2002 se estableció en Bolivia la Ley del Seguro Universal Materno Infantil $(\text { LSUM })^{20}$. Esta ley incluye el diagnóstico y el tratamiento oportuno y gratuito de sífilis materna y neonatal. Diez años después de las estimaciones que se realizaron en Cochabamba, Bolivia y dada la disponibilidad del LSUMI en el nivel nacional, surge la hipótesis de que la prevalencia de sífilis materna disminuyó significativamente respecto de la única estimación registrada en el HGU. El presente trabajo tuvo por objeto a) evaluar la prevalencia de sífilis materna en el HGU, b) evaluar la utilidad de una prueba rápida treponémica y c) estudiar las características de las mujeres asociadas a la prevalencia de sífilis materna.

\section{MÉTodos}

Se realizó un estudio transversal que incluyó mujeres que fueron atendidas de parto en el HGU en la ciudad de Cochabamba, Bolivia, entre marzo y abril de 2003. Las mujeres que participaron en el estudio firmaron un consentimiento informado sobre los objetivos del mismo y contestaron un cuestionario que indagó características socioeconómicas y demográficas como la edad, escolaridad, estado civil, ocupación, recuento de bienes en el domicilio y tiempo de trayecto del domicilio 
al hospital. Se indagó también sobre antecedentes ginecobstétricos como el número de partos, número y tipo de abortos, número de consultas de control prenatal, pruebas de sífilis realizadas durante las consultas, el conocimiento de la gratuidad de los servicios y el antecedente de infecciones de transmisión sexual (ITS). El protocolo del trabajo fue evaluado por el Comité de Ética del Instituto Nacional de Salud Pública de México y por las autoridades del HGU.

Las mujeres proporcionaron $10 \mathrm{ml}$ de sangre por venopunción. Se utilizó una gota de sangre completa para la realización de la prueba rápi$\mathrm{da}^{17}$. Se realizó la prueba de RPR (reaginas rápidas en plasma) en los Laboratorios de Investigación Médica (LABIMED) de la Universidad Mayor de San Simón en Cochabamba. Los sueros positivos a las pruebas Determine y RPR fueron sometidos a la prueba confirmatoria FTA-ABS. Resultados reactivos a las pruebas de RPR y FTAABS definieron los casos de sífilis materna. Las mujeres que cumplieron con la definición de caso y también las que resultaron positivas a las pruebas Determine y FTA-ABS recibieron tratamiento consistente con 2,4 millones de UI de penicilina benzatínica por vía intramuscular una vez por semana por tres semanas consecutivas. Los hijos de estas mujeres que resultaron con pruebas FTA-ABS IgM positivas, recibieron tratamiento con penicilina procaínica $(50.000 \mathrm{UI} / \mathrm{kg}$ por día) por vía intramuscular por día por 10 días. La prueba FTA-ABS sirvió como referencia para calcular los parámetros de sensibilidad, especificidad y valores predictivos de los resultados positivo y negativo de la prueba Determine.

Siguiendo el procedimiento propuesto por Briones $^{21}$, se construyó un índice de nivel socioeconómico que incluyó tres componentes (hacinamiento, bienes en el hogar y nivel de educación). Cada componente fue dividido en tres categorías: alto, medio y bajo. Se realizó una validación interna del índice al cruzarlo con cada uno de sus componentes y calculando la prueba de gamma para variables ordinales. Se realizó también una validación externa cruzando el índice de nivel socioeconómico con indicadores que no fueron incluidos en la construcción del índice como televisión y lavadora. Se obtuvieron los siguientes coeficientes de gamma por cada componente respecto del índice: $0,77,0,86,0,89$ para hacinamiento, bienes en el hogar y nivel de educación, respectivamente. Para la validación externa los coeficientes de gamma fueron: $0,72 \mathrm{y}$ 0,58 para lavadora y televisión, respectivamente.

A fin de estimar la magnitud de la asociación entre la seroprevalencia de sífilis materna y las características de las mujeres se calculó el odds ratio de la prevalencia con sus correspondientes intervalos de confianza y valores de p. Se realizó un análisis multivariado por regresión logística para observar si los coeficientes de las variables utilizadas en el modelo saturado eran estadísticamente significativos. El modelo estuvo constituido por las variables que en el análisis crudo tuvieron en al menos uno de sus estratos una relación significativa con la prevalencia de sífilis materna $(p<0,05)$, con la excepción de la variable nivel de escolaridad que estaba implícita en el índice de nivel socioeconómico.

\section{Resultados}

Se estudiaron 489 mujeres en el HGU quienes tuvieron en promedio 24 años de edad (rango 1544). En su mayoría eran mujeres jóvenes entre $15 \mathrm{y}$ 25 años (64,4\%), amas de casa (68,1\%), habían terminado la educación básica $(54,6 \%)$ y fuemon clasificadas en el nivel socioeconómico bajo (60,7\%). Aunque un poco más de la mitad de las mujeres vivían en concubinato $(55,2 \%)$, la gran mayoría tenía pareja estable (88,1\%). Las mujeres iniciaron su vida sexual a los 17 años en promedio (rango 12-33). El antecedente autorreferido de ITS fue de 5\%. El número de embarazos fue de 3 en promedio (rango $1-12)$ y casi un tercio del total de mujeres (30,5\%) refirió haber tenido al menos un aborto.

Aunque la gran mayońa de las mujeres refirió haber tenido al menos una consulta prenatal (81\%, $396 / 489$ ), sólo $15 \%$ de ellas refirí que le habían practicado la prueba de sífilis $(59 / 396)$ y de éstas últimas en 59,3\% (35/59) refieren haber recibido el resultado. Sólo 72 mujeres del total $(14,7 \%)$ estaban informadas de que la prestación de servicios de consulta prenatal y atención de parto, incluidas las pruebas antitreponémicas y el tratamiento con penicilina, eran gratuitos en el hospital.

De las 489 mujeres estudiadas, 22 resultaron positivas tanto a la prueba de RPR como a la de FTAABS dando una prevalencia de sífilis materna de

ARtíCULO DE 
4,5\%. Sin embargo, 50 mujeres fueron positivas a las pruebas Determine y FTA-ABS, dando una prevalencia de 10,2\%. La evaluación de la prueba Determine mostró sensibilidad, especificidad, valores predictivos de los resultados positivo y negativo de $98 \%$, 99,8\%, 98\% y 99,8\%, respectivamente (Tabla 1).

Los resultados del análisis bivariado presentados en la Tabla 2 mostraron que hubo una tendencia significativa entre el incremento de la prevalencia de sífilis materna y el incremento de la edad y también del número de embarazos (en el grupo de menor edad la prevalencia fue de $6,4 \%$ en contraste con $16,7 \%$ en el de mayor edad, mientras que en las mujeres con un embarazo fue de $6,5 \%$, en aquellas con tres y más fue de 13,7\%). Por el contrario, a menores niveles socioeconómico y de escolaridad, mayor prevalencia de infección por sífilis (en los niveles socioeconómico alto y escolaridad alta las prevalencias fueron de $2,4 \%$ y 4,2\%, mientras que en los niveles bajos fueron $14,5 \%$ y $19,5 \%$, respectivamente). Se encontró que las mujeres que refirieron estar unidas como concubinas tuvieron un riesgo significativamente mayor de sífilis (OR 2,8, IC95\% $1,0-8,0)$ respecto de las mujeres solteras, pero entre éstas y las casadas no difinieron en la magnitud de la infección. El riesgo de infección por síflis fue significativamente mayor entre las mujeres que refirieron tener antecedentes de ITS (OR 4,9, IC 95\% 2,0-12,2) que aquellas que no lo refirieron y también entre aquellas que tardaron más tiempo en llegar desde su domicilio al hospital (OR 2,7, IC 95\% 1,1$6,4)$ respecto de las que tardaron menos tiempo. La magnitud de la infección por sífilis no tuvo relación con la ocupación de las mujeres. Las caractenísticas de las mujeres que estuvieron asociadas en forma independiente con la infección por sífilis fueron la edad, el índice de nivel socioeconómico y el antecedente de ITS. Aunque hubo una relación directamente proporcional entre el incremento de riesgo de infección por sífilis y el incremento de la edad, sólo el grupo de mayor edad tuvo un riesgo estadísticamente mayor respecto del grupo de referencia (OR 4,5, IC 95\% 1,5-13). En forma inversa, sólo las mujeres pertenecientes a la categoría baja del índice de nivel socioeconómico tuvieron un riesgo significativamente mayor de infección por sífilis respecto de aquellas pertenecientes a la categonía alta del índice (OR 8,5, IC 95\% 1,1-67).

\section{DisCUSIÓN}

En el presente trabajo se encontró una prevalencia de sífilis materna en el HGU de 4,5\%, similar a la prevalencia global de sífilis materna de 4,3\% reportada para Bolivia en $1996^{13}$. Sin embargo, aquella cifra es menor a la reportada previamente para el HGU de 10,9\% en $1996^{13}$. Este resultado apoya la hipótesis de que en 10 años ha habido una disminución significativa de la prevalencia de sífilis materna entre las mujeres que acuden a atenderse de parto en el HGU. No obstante, la prevalencia de sífilis materna de $4,5 \%$ sigue siendo alta en el contexto epidemiológico de sífilis materna en América Latina y el Caribe y sugiere que debe mejorarse el sistema de vigilancia de sífilis materna en el HGU para abatir dicha magnitud "operacionalizando" correctamente la LSUM.

\section{Tabla 1. Validación de las pruebas D etermine T P respecto de la prueba FTA-ABS en el H ospital Germán Urquidi en Cochabamba, Bolivia en 2003}

\begin{tabular}{|c|c|c|c|c|c|c|c|}
\hline Pruebas & & $\begin{array}{c}\text { FTA-ABS } \\
(+) \\
n=51\end{array}$ & $\begin{array}{c}\text { FTA-ABS } \\
(-) \\
n=438\end{array}$ & Sensibilidad & Especificidad & VPRPb & VPRN $^{\mathrm{C}}$ \\
\hline Determine TP & $\begin{array}{l}+ \\
-\end{array}$ & $\begin{array}{c}50 \\
1\end{array}$ & $\begin{array}{c}1 \\
437\end{array}$ & 98,0 & 99,8 & 98,0 & 99,8 \\
\hline
\end{tabular}

aPrueba de FTA-ABS

bValor predictivo del resultado positivo

cValor predictivo del resultado negativo 
Tabla 2. C aracterísticas de las mujeres relacionadas con la prevalencia de sífilis materna en el H ospital Germán U rquidi en Cochabamba, Bolivia en 2003

\begin{tabular}{|c|c|c|c|c|c|c|}
\hline C aracterísticas & $\mathbf{n}$ & Prevalencia & $P O R^{a}$ & CI 95\% & $P O R^{b}$ & CI 95\% \\
\hline \multicolumn{7}{|l|}{ Edad } \\
\hline $15-20$ & 157 & 6,4 & 1,0 & & 1,0 & \\
\hline $21-25$ & 158 & 8,9 & 1,4 & $0,6-3,3$ & 1,2 & $0,5-3,0$ \\
\hline $26-30$ & 90 & 14,4 & 2,5 & $1,0-5,9^{c}$ & 2,1 & $0,7-6,1$ \\
\hline $31-44$ & 84 & 16,7 & 2,9 & $1,2-6,9^{c}$ & 4,5 & $1,5-13^{c}$ \\
\hline \multicolumn{7}{|l|}{ Índice de NSE } \\
\hline Alto & 84 & 2,4 & 1,0 & & 1,0 & \\
\hline Medio & 108 & 5,6 & 2,4 & $0,5-12,3$ & 3,6 & 0,4-32 \\
\hline Bajo & 297 & 14,5 & 6,9 & $1,6-29,3^{c}$ & 8,5 & $1,1-67^{c}$ \\
\hline \multicolumn{7}{|l|}{ Escolaridad } \\
\hline Alta & 118 & 4,2 & 1,0 & & & \\
\hline Medio & 267 & 9,7 & 2,4 & $0,9-6,5$ & & \\
\hline Baja & 104 & 19,2 & 5,4 & $1,9-14,9$ c & & \\
\hline \multicolumn{7}{|l|}{ Estado civil } \\
\hline Soltera & 76 & 5,3 & 1,0 & & 1,0 & \\
\hline Casada & 143 & 7,7 & 1,5 & $0,5-4,9$ & 0,4 & $0,1-2,0$ \\
\hline Concubina & 270 & 13,3 & 2,8 & $1,0-8,0^{\mathrm{C}}$ & 1,2 & $0,3-4,6$ \\
\hline \multicolumn{7}{|l|}{ Ocupación } \\
\hline Estudia & 36 & 2,8 & 1,0 & & 1,0 & \\
\hline Trabaja & 120 & 6,7 & 2,5 & $0,3-20,7$ & 0,4 & $0,1-4,6$ \\
\hline Ama de casa & 333 & 12,6 & 5,1 & $0,7-37,8$ & 1,4 & $0,5-13,7$ \\
\hline \multicolumn{7}{|c|}{ Antecedente de ITS } \\
\hline No & 456 & 9,2 & 1,0 & & 1,0 & \\
\hline Sí & 24 & 33,3 & 4,9 & $2,0-12,2^{c}$ & 4,1 & $1,2-13^{c}$ \\
\hline \multicolumn{7}{|c|}{ Número de embarazos } \\
\hline Uno & 154 & 6,5 & 1,0 & & 1,0 & \\
\hline Dos & 131 & 9,9 & 1,6 & $0,7-3,7$ & 1,2 & $0,4-3,5$ \\
\hline Tres y más & 204 & 13,7 & 2,3 & $1,1-4,9^{c}$ & 1,1 & $0,4-3,7$ \\
\hline \multicolumn{7}{|c|}{ Tiempo en trayecto al hospital } \\
\hline$<30$ minutos & 308 & 10,4 & 1,0 & & 1,0 & \\
\hline 31-60 minutos & 147 & 7,5 & 0,7 & $0,3-1,4$ & 0,5 & $0,2-1,2$ \\
\hline$>61$ minutos & 34 & 23,5 & 2,7 & $1,1-6,4^{c}$ & 1,7 & $0,6-4,7$ \\
\hline
\end{tabular}

aOdds ratio crudo razón de momios cruda.

bodds ratio ajustado razón de momios ajustada.

c $p<0,05$.

Aunque la LSUMI propone la prestación de servicios de atención prenatal y de parto gratuitos, incluyendo diagnóstico y tratamiento sifilíticos, los resultados mostraron que esos servicios no son aplicados a toda la población que busca atención prenatal y atención de parto en el HGU. En este trabajo, $81 \%$ de las mujeres estudiadas refinó haber tenido al menos una consulta prenatal en dicho hospital pero sólo 15\% de ellas refinó que se les había realizado la prueba de sífilis. De estas últimas, 40,7\% no recibieron el resultado de la prueba. Estos resultados concuerdan con el análisis realizado por otros autores sobre sífilis matema y congénita en Bolivia en que encontraron un sistema de detección y tratamiento de sífilis matema y congénita deficiente, a pesar del establecimiento de la $\mathrm{LSUM}^{22}$. Cuando se 
preguntó a las mujeres sobre si tenían conocimiento sobre la gratuidad de los servicios de consulta prenatal y atención de parto, incluidas las pruebas diagnósticas de sífilis y el tratamiento, sólo $14 \%$ de ellas respondieron afirmativamente lo cual denota que dichas condiciones de gratuidad de los servicios son conocidas por una proporción pequeña de mujeres que buscan atención prenatal y obstétrica en el HGU.

Por otra parte, la prueba rápida Determine tiene ventajas que muestran su utilidad como prueba de tamizaje para sífilis materna en América Latina como: a) su uso individual en el lugar de la consulta, b) su aplicación no necesita de una infraestructura de laboratorio, luz eléctrica y refrigeración y c) el resultado se tiene en sólo $15 \mathrm{~min}$. Asimismo, está disponible comercialmente en Latinoamérica y su costo no es excesivamente alto pues se ha reportado que en Bolivia asciende a 1,48 dólares ${ }^{23}$. En el presente estudio Determine tuvo una sensibilidad de 98\% y una especificidad de 99,8\% usando sangre total y utilizando la prueba FTA-ABS como referencia. Aunque los fabricantes comunican una sensibilidad de $92 \%$ y una especificidad de $100 \%$ cuando se usa sangre total ${ }^{17}$, en el presente trabajo la sensibilidad no se vio afectada por el uso de sangre total. La principal limitación de la prueba Determine, en contraste con las pruebas RPR o VDRL, estriba en que detecta anticuerpos específicos contra $\mathrm{T}$ pallidum que tienden a persistir por años en la sangre de la persona infectada y puede no distinguir por ello entre sífilis activa y sífilis latente. Es por ello que la magnitud de la prevalencia calculada con los resultados de la prueba Determine/FTA-ABS fue un poco mayor del doble que con la prueba RPR/FTA-ABS, lo cual muestra un sobrediagnóstico de sífilis usando Determine. No obstante, el uso de pruebas rápidas para el control de sífilis congénita debe ponerse en la perspectiva de la salud pública en países con alta prevalencia de sífilis materna, como Bolivia. Tinajeros y cols $^{24}$ han discutido que el uso sistemático de pruebas rápidas en mujeres embarazadas en Bolivia permite la detección de hasta $92 \%$ de casos de sífilis materna y sus correspondientes casos de sífilis neonatal mientras que con el sistema tradicional de pruebas como RPR realizadas en el laboratorio del hospital, solamente se detecta aproximadamente 38\% de los casos de sífilis materna. En el caso del sobrediagnóstico de sífilis materna por la utilización de la prueba rápida, la aplicación de un nuevo tratamiento con penicilina a una mujer embarazada con historia de sífilis puede justificarse al no tener la certeza de que esa mujer pudo haber tenido en el pasado un tratamiento incompleto o haber sufrido reinfección por T pallidum.

Respecto a las caractenísticas de las personas asociadas independientemente con la infección se encontró que, respecto de la edad, el riesgo de infección fue significativo sólo para el gnupo 34 a 40 (OR =1.5, IC95\% 4.5-13), tomando como referencia el grupo de menor edad. Este resultado contrasta con lo reportado al respecto por diferentes autores en Brasil donde el riesgo de infección por sífilis fue mayor entre las mujeres más jóvenes, con edad menor a 17 años ${ }^{8}$ y a 23 años ${ }^{14}$. Asimismo, los resultados en este estudio sugieren que las mujeres con menor nivel socioeconómico (evaluado por el índice construido) tendrían menor acceso a la educación formal y al mismo tiempo menos recursos económicos para solventar sus necesidades en salud. Ello haría a estas mujeres más susceptibles a la infección por sífilis que a las mujeres con mayor nivel socioeconómico (OR $=8,5$, IC 95\% 1,1-67). Dos estudios realizados en Brasil mostraron que un ingreso menor de 1 salario mínimo o menor a 30 dólares norteamericanos (indicadores que reflejan un nivel socioeconómico bajo) estuvieron asociados significativamente con la presencia de la infección ${ }^{8,14}$. En Bolivia se encontró que una baja escolaridad y el no haber visto televisión (por no tenerla) durante las dos últimas semanas previas a la entrevista, estuvieron asociadas en forma independiente con la prevalencia de sífilis matema ${ }^{13}$. Finalmente, la mayor frecuencia de sífilis matema entre las mujeres que tuvieron el antecedente de ITS sugiere que la transmisión de la infección estuvo relacionada con un comportamiento sexual de riesgo, ya sea de las mujeres o de sus parejas sexuales.

En el presente trabajo se concluye que la prevalencia de sífilis matema encontrada en el HGU en Cochabamba, Bolivia, es alta en el contexto de la epidemiología de la sífilis matema en América Latina por lo que se sugiere reforzar la aplicación de la LSUMI en un programa de vigilancia epidemiológica de sífilis materna en el hospital objeto de estudio para abatir dicha magnitud en el mediano plazo. Asimismo, se sugiere el uso sistemático de pruebas rápidas para la detección del mayor número posible de casos de sífilis matema y la aplicación de tratamiento oportuno a fin de evitar casos de sífilis neonatal. Características de nivel socioeconómico bajo y prácti- 
cas sexuales de riesgo siguen siendo los principales factores de riesgo de infección de sífilis matema en la población bajo estudio.

\section{REFERENCIAS}

1. MuLck S, Broutet N, Htun Y, Temmerman M, Ndowa F. Controlling congenital syphilis in the era of HIV/AIDS. Bull World Health Organ 2004; 82: 431-2.

2. Radolf JD, SÁnchez PJ, Schulz KF, MuRphy FK. Congenital syphilis. En: Holmes KK, Sparling PF, Mårdh P, Lemon SM, Stamm WE, Piot P, Wasserheit JN, Editors. Sexually Transmitted Diseases. 3a ed. New York: McGraw Hill; 1999; 1165-89.

3. Watson-Jones D, Changalucha J, Gumodoka B, Weiss H, Rusizoca M, NDEKI L ET AL Syphilis in pregnancy in Tanzania. I. Impact of maternal syphilis on outcome of pregnancy. J Infect Dis 2002; 186: 940-7.

4. VALDERRAMA J, ZACARIAS F, MAZIN R. Śfilis matema y sífilis congénita en Aménica Latina: un problema grave de solución sencilla. Rev Panam Salud Publica 2004; 16: 211-17.

5. Behest FM, Desomeaux J, Joseph D, Adrien M, Colcou G, DaLABEtTa G ET AL. Control of sexually transmitted diseases in Haiti: results and implications of a baseline study among women living in Cité Soleil Shantytowns. J Infect Dis 1995; 172: 764-71.

6. FttZgeraid DW, Behest FMT, LUCEt C, Roberfroid D. Prevalence, burden and control of syphilis in Haiti's nural Artibonite region. Int J Infect Dis 1998; 2: 127-31.

7. Jacquier N, Dos Santos L, Deschutter JD, Duarte B, Rodríguez M, Martineu M et aL Syphilis in adolescent mothers in the city of Posadas, Province of Misiones. Medicina (B Aires) 1999; 59: 437-45.

8. Rodríguez CS, GuimarÃES MDC. Positividade para sífilis em puérperas: ainda um desafio para o Brasil. Rev Panam Salud Publica 2004; 16: 168-75.

9. Hernández-Trejo M, Hernández-Prado B, Uribe-Salas F JuÁrEZ-Figueroa L, CONDE-GonZÁIFZ CJ. Sífilis matema y congénita en dos hospitales mexicanos: evaluación de una prueba diagnóstica rápida. Rev Invest Clin 2006; 58: 119-25.

10. Noyola DE, Malacara-Aifaro O, Lma-Rogel V, TorresMontes A. Seroprevalencia de sífilis en mujeres embarazadas en San Luis Potosí. Salud Publica Mex 2006; 48: 151-4.

11. Lumbiganon P, Piaggio G, Vimar J, Pinol A, Bakketeig L, BeRgSJO $P$ ET AL The epidemiology of syphilis in pregnancy. Int J STD \&AIDS 2002; 13: 486-94.

12. Scott-Wright A, Hakre S, Bryan JP, Jaramilo R, Reyes LG, Cruess D et al. Hepatitis B virus, Human immunodeficiency virus type-1, and syphilils among women attending prenatal clinics in Belize, Central America. Am J Top Med Hyg 1997; 56: 285-90.
Agradecimientos

N Villazón-Vargas fue estudiante de maestría en Ciencias de la Salud en el Instituto Nacional de Salud Pública donde se graduó el 26 de julio de 2005.

13. Southwick KL, Bianco S, Santander A, Estenssoro M, Torrico F, SEOANe G ET aL Maternal and congenital syphilis in Bolivia, 1996: prevalence and risk factors. Bull World Health Organ 2001; 79: 33-42.

14. Lago EG, Rodríguez LC, Fiori RM, Stein AT. Congenital sífilis. Identification of two distinct profiles of matemal characteristics associated with risk. Sex Transm Dis 2004; 31: 33-7.

15. Trepka MJO, Bloom SA, Zhang G, Kim S, Nobies RE. Inadequate syphilis screening among women with prenatal care in a community with high syphilis incidence. Sex Transm Dis 2006; 33: 670-4.

16. Worid Health Organization. The use of rapid syphilis test. Zwitzenland, WHO/TDR, 2006. Disponible en: http://www.who.int/std_diagnostics/publications/manuals/Syphilis_Eng_14May07.pdf (página consultada el día 20 de noviembre de 2008).

17. Аввотt Laboratories. Determine syphilis TP test for antiTreponema pallidum antibodies in senum or plasma or whole blood. Troubleshooting guide, 1998.

18. The sexually transmitted diseases diagnostics initiative. Laboratory-based evaluation of rapid syphilis diagnostics. Zwitzenland, UNDP/World Bank/WHO, 2003. Disponible en: http://www.who.int/std diagnostics/ publications/meetings/SDI Report.pdf (página consultada el día 20 de noviembre de 2008).

19. Jú́rez-Figueroa L, Uribe-Salas F, García-Cisneros S, OlaMENDI-Portugal M, Conde-Giez CJ. Evaluation of a rapid strip and a particle agglutination test for syphilis diagnosis. Diagn Microbiol Infect Dis 2007; 59: 123-6.

20. Ley del Seguro Universal Matemo Infantil en Bolivia. Disponible en: http://www.gparlamentario.org/pdf/ Bolivian\%20Legislation/Ley\%20SUMI.pdf (página consultada el día 10 de febrero de 2008).

21. BRIONES G. Métodos y técnicas de investigación para las ciencias sociales. $3^{2}$ ed. México: Trillas; 1998; 192-4.

22. Deperthes BD, Merheus A, O’ReiuY K, Broutet N. Matemal and congenital syphilis programmes: case studies in Bolivia, Kenya and South Africa. Bulletin of the World Health Organization 2004; 82: 410-16.

23. Levin CE, Steele M, Atheriy D, García SG, Tinajeros F, REVOwO R, Richmond K ET AL. Analysis of the operational costs of using rapid syphilis test for detection of matemal syphilis in Bolivia and Mozambique. Sex Transm Dis 2006; 33: 1-8.

24. Tinajeros F, Grossman D, Richmond K Steele M, García SG, ZegarRa L, Revowo R. Diagnostic accuracy of a point-of-care syphilis test when used among pregnant women in Bolivia. Sex Transm Infect 2006; 82 (suppl V): V17-V21.

ARtíCULO DE 